\title{
Real-Time SPECT and 2D Ultrasound Image Registration
}

\author{
Marek Bucki ${ }^{1}$, Fabrice Chassat $^{2}$, Francisco Galdames ${ }^{3}$, Takeshi Asahi ${ }^{2}$, \\ Daniel Pizarro ${ }^{2}$, and Gabriel Lobo ${ }^{4}$
}

1 TIMC Laboratory, UMR CNRS 5225, University Jospeh Fourier, 38706 La Tronche, France

Marek.Bucki@imag.fr

${ }^{2}$ Center of Mathematical Modeling, UMI CNRS 2807, University of Chile, Blanco Encalada 2120, Santiago, Chile

${ }^{3}$ Electrical Engineering Department, University of Chile, Tupper 2007, Santiago, Chil

${ }^{4}$ Nuclear Medicine Service, San-Juan de Dios hospital, Occidental metropolitan service health, Huerfanos 3255, Santiago, Chile

\begin{abstract}
In this paper we present a technique for fully automatic, realtime 3D SPECT (Single Photon Emitting Computed Tomography) and $2 \mathrm{D}$ ultrasound image registration. We use this technique in the context of kidney lesion diagnosis. Our registration algorithm allows a physician to perform an ultrasound exam after a SPECT image has been acquired and see in real time the registration of both modalities. An automatic segmentation algorithm has been implemented in order to display in 3D the positions of the acquired US images with respect to the organs.
\end{abstract}

\section{Introduction}

Nowadays medical images are classically used to help physicians achieve more accurate diagnosis. In the literature, 1] have defined basic criteria to classify all types of medical image registrations. We focus on $2 \mathrm{D} / 3 \mathrm{D}$ intrasubject multimodality registration, which means that we consider two modalities of images for the same patient at almost the same moment: functional 3D SPECT and anatomical 2D US images. This choice is motivated by the diagnosis we want to improve and the fact that these low cost technologies are available in most of the medical centers in Chile, where this study took place.

The system is intended to help the surgeon differentiate acute lesions from scars in urinary tract infections. A discordant result between SPECT and US may occur because: 1) a lesion is seen in one but not in the other image (acute lesions) or 2) the US exploration simply missed a zone. Image registration provides better correlation between SPECT and US results. The superimposition of both modalities helps overcome that issue by visualizing the data in a common referential.

A great amount of work can be found dealing with this registration problem, however in the case of 3D SPECT and 2D US registration for diagnosis, most of it is not applicable. A major drawback of the technique proposed in 2 is that it only matches a few selected US images with the SPECT volume. The purpose of

N. Ayache, S. Ourselin, A. Maeder (Eds.): MICCAI 2007, Part II, LNCS 4792, pp. 219 226, 2007.

(C) Springer-Verlag Berlin Heidelberg 2007 
our registration system is to locate on a US image an organ area which has been identified in the SPECT volume. This is totally incompatible with the selection of a few ultrasound images.

Extrinsic registration methods often need invasive markers or fiducials [3] [4] which is also incompatible with non-invasive diagnosis. As for intrinsic registration method, we face the problem of using two different image modalities, which makes it extremely difficult to find common landmarks. Some intrinsic registration can be made by common segmentation of the same object and distance minimization between the segmented points or surfaces [5] 6]. This method is quite inappropriate in the case of US, as automatic segmentation of kidney is still an issue.

Mutual information based registration [7] could be of interest, nevertheless we decided not to go further in this direction, as the common base needed for this method seems hard to find in the case of US, most of the documented studies being based on MRI or CT 8].

In order to overcome the issues mentioned above, our registration strategy is based upon the calibration of both scanners (SPECT and US) using a calibration frame and a localizer. This method [9] allows us to obtain easily the rigid transformation between the SPECT and US referentials but requires that the patient remains still during the whole procedure: SPECT acquisition followed by the US exam. Our system is implemented on a Surgetics Station ${ }^{\circledR}$ provided by Praxim (France). This station is equipped with a Polaris ${ }^{\circledR}$ infra-red optical passive localizer, manufactured by Northern Digital Inc. (Canada) which allows us to track in space so-called 'rigid bodies'.

We fix a physical reference 'rigid body' on the gamma camera bed. Assuming that the patient does not move during the whole exam, this bed reference represents also the patient space. Another rigid body is fixed on the transducer in order to track the US images. Once both US and SPECT images' positions have been converted into the common patient referential we extract from the SPECT volume the slices corresponding to the US images. The resulting composite image, comprising both anatomical and functional information, is displayed on the station screen in real-time during the US exam. In addition, our software implements an automatic segmentation algorithm of 3D SPECT images, that lets the physician navigate the US image position with respect to the organ (see section 4).

\section{Registration Overview}

In order to merge SPECT and US data we need to compute for every US image pixel the corresponding SPECT value. This value is interpolated from the voxels closest to the physical US pixel position within the patient SPECT volume.

We define the following referentials: $U S$ for the US image, $P R O B E$ for the US probe, $L O C$ for the station localizer, BED for the gamma camera bed, MIRE for the calibration cube and SPECT for the SPECT volume.

The matrix that gives the position of any US pixel $P$ within the SPECT volume is $M_{U S \rightarrow S P E C T}$ such as: $(P)_{S P E C T}=M_{U S \rightarrow S P E C T} *(P)_{U S}$. This 


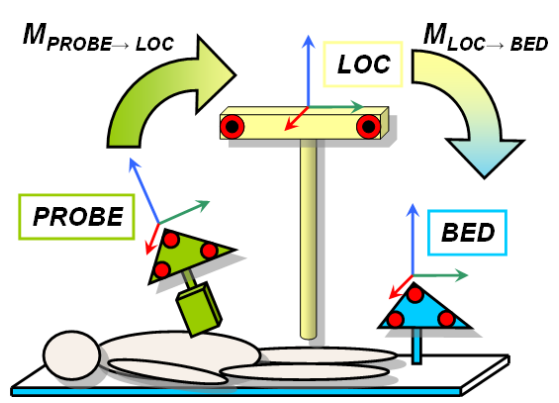

(a)

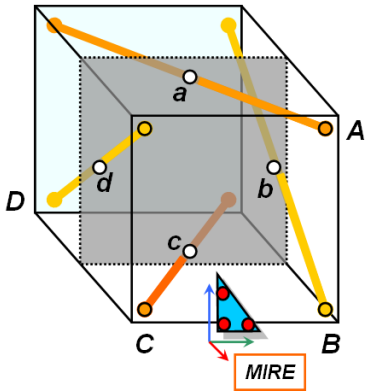

(b)

Fig. 1. (a) Referentials being tracked by the system during image registration. (b) Calibration cube with the 4 catheters labelled A, B, C, D and the corresponding 'stains' a, b, c and d on a SPECT Z-slice (grey square).

matrix can be written as: $M_{U S \rightarrow S P E C T}=M_{B E D \rightarrow S P E C T} * M_{L O C \rightarrow B E D} *$ $M_{P R O B E \rightarrow L O C} * M_{U S \rightarrow P R O B E}$.

The time consuming calibration procedures are carried out beforehand. In order to compute $M_{U S \rightarrow P R O B E}$ we use the well-tried freehand membrane scan method [10. Section 3 describes the procedure to obtain $M_{B E D \rightarrow S P E C T}$. These calibration matrices remain valid for all subsequent exams, as explained below.

During the exam, the station localizer computes both $M_{L O C \rightarrow B E D}$ and $M_{P R O B E \rightarrow L O C}$ (see Fig. 1a). The $M_{U S \rightarrow S P E C T}$ matrix is assembled and for each pixel in the US image the corresponding SPECT value is computed by trilinear interpolation within the SPECT volume. A color code is associated with the pixel and the resulting color image is blended with the original US image. The physician can select the level of transparency to put emphasis on anatomical or physiological information.

\section{SPECT Calibration}

To compute $M_{B E D \rightarrow S P E C T}$ we use a calibration cube 9]. A rigid body is mounted on its front side defining the MIRE referential. Inside the cube 4 catheters filled with technetium run along the faces (see Fig. 10). The cube is roughly positioned parallel to $Z_{S P E C T}$ axis, i.e. the bed axis, and scanned. The catheters appear clearly within the SPECT volume (see Fig. 2a).

The catheters are segmented as follows. An initial threshold level is chosen and all voxels with lower intensity are ignored. From each Z-slice of the volume we extract a set of connex areas of pixels having an appropriate aspect ratio, that we call 'stains'. We compute the gravity center of the 4 stains with the highest maximal greylevel. Ideally these gravity centers form a square but since the cube can be misaligned with $Z_{S P E C T}$, we admit some tolerance around the threshold aspect ratio used to accept or reject the 'square' formed by the 4 stain centers. Once all the slices have been processed, the 4 sets of valid stain centers 


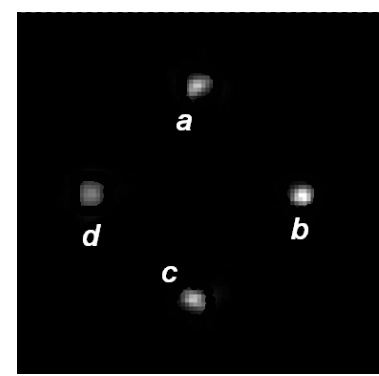

(a)

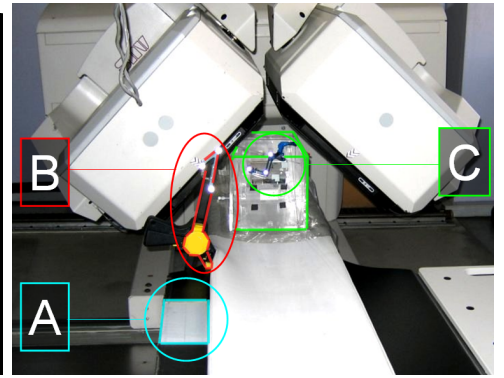

(b)

Fig. 2. (a) SPECT Z-slice of a cube. The catheters generated the 'stains' a, b, c and d (see also Fig. 10). (b) Set-up for SPECT calibration. A: plate referential permanently fixed on the bed. B: $B E D$ rigid body fixed on the bed for the duration of the exam. C: $M I R E$ rigid body fixed on the calibration cube.

are linked together using a proximity search to form 4 point clouds, one for each catheter. Then a Principal Component Analysis is carried out on each point cloud to find its main inertial axis (i.e. the catheter). After these geometrical features have been extracted, they are labelled A, B, C and D using a-priori knowledge of the SPECT referential orientation. The current segmentation error is computed as the maximum distance between all stain centers and their corresponding catheter. The initial threshold is increased and this procedure is repeated as long as a minimum of 40 slices can be properly segmented. The retained segmentation is the one with the minimal error.

Once the 4 catheters have been segmented in $S P E C T$, we build an intermediary referential $L O G$. The center of $L O G$ is the point $O_{L O G}$ that minimizes the distance to the 4 catheter axes. It is obtained by simple geometrical construction. We use this point along with its projections on axes $A$ and $B$ (see Fig. 10) to define the referential $X_{L O G}$ and $Y_{L O G}$ unitary vectors. The $Z_{L O G}$ vector is obtained by cross product.

We proceed in the same way to build the $L O G$ referential in MIRE. This time we use a pointer with a rigid body mounted on it to localize the tips of the 4 catheters in the physical space of MIRE. This operation needs not to be done each time we calibrate the gamma camera. We call it 'calibration of the calibration'.

We use the $L O G$ referential to assemble $M_{M I R E \rightarrow L O G}$ and $M_{L O G \rightarrow S P E C T}$. Then the localizer retrieves the position of $M I R E$ with respect to $B E D$ and finally the SPECT calibration matrix is computed as:

$M_{B E D \rightarrow S P E C T}=M_{L O G \rightarrow S P E C T} * M_{M I R E \rightarrow L O G} * M_{B E D \rightarrow M I R E}$.

This matrix is valid as long as we keep the $B E D$ reference mounted on the bed and needs not to be recomputed for the subsequent patients. For practical reasons though, instead of leaving a rigid body permanently fixed to the bed, we chose a more robust solution to store our calibration matrices. We mounted on the bed side a $20 \times 10 \times 1 \mathrm{~cm}$ thick plate having 5 calibrated holes on its surface. 
Unlike the $B E D$ rigid body, this plate does not interfere with the classical use of the gamma camera and can be left in place (cf. Fig. $2 \mathrm{~b}$ ). The reference system defined by this plate is recovered each time a new rigid body is fixed on the bed. To this end a pointer is used to localize the 5 holes in space. The SPECT calibration matrix is then transferred from the plate coordinates system into the bed referential.

Finally we store in our system several calibrations made with distinct gamma camera parameters. At the moment of the exam, the physician chooses the calibration that fits the patient morphology.

\section{Segmentation}

To perform the segmentation we chose a deformable model method for its robustness and high noise immunity [11. Our model is a simplex mesh that is iteratively adjusted to the form of the kidney. Simplex meshes are appropriate for this type of modeling since the position of a vertex can be expressed as a function of the position of its three neighboring vertices and the shape parameters 12 13. and because the deformation is controlled by discrete geometrical entities, allowing a simple control.

In order to obtain an initial simplex mesh, we first generate a triangle mesh using a 'marching cubes' algorithm [14. By applying a topological dual operation to the triangle mesh of this initial isosurface of the kidneys we obtain the initial simplex mesh of our model.

The iterative deformation of the model is controlled by means of a Newtonian law of motion, using internal and external forces [15]:

$$
m \frac{\partial^{2} P_{i}}{\partial t^{2}}=-\gamma \frac{\partial P_{i}}{\partial t}+\vec{F}_{i n t}+\vec{F}_{e x t}
$$

where $m$ is the mass of a vertex, usually $1, P_{i}$ is the position of the vertex $i, \gamma$ is the damping factor and $\vec{F}_{i n t}, \vec{F}_{\text {ext }}$ are the internal and external forces. The external forces push the vertices towards the edges of the kidney and are derived from the gradient $\nabla f(x, y, z)$ of the edge map $f=|\nabla \operatorname{Image}(x, y, z)|^{2}$ of the image, computed with a Sobel filter. The internal forces are given by the model 12] 13. and their purpose is to control the smoothness of the deformation. The iterations stop when the mean deformation of the mesh is smaller than $0.1 \%$.

\section{Clinical Protocol}

The registration protocol is carried out in the following way. First, a $B E D$ rigid body is mounted on the bed and its position localized within the bed plate referential. Then the patient is positioned and prepared for the SPECT exam in a classical way. The SPECT is acquired. The acquisition parameters along with the data volume are loaded into the Surgetics Station ${ }^{\circledR}$ and the volume segmentation is carried out. The patient stays on the bed without moving. A 


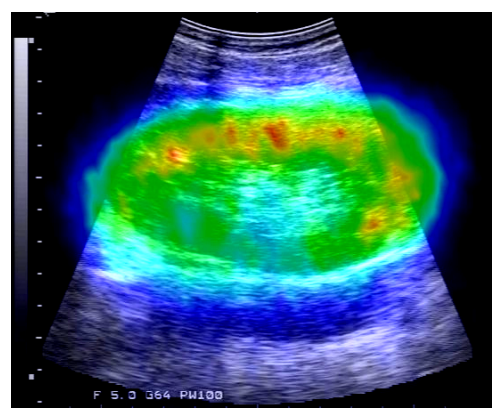

(a)

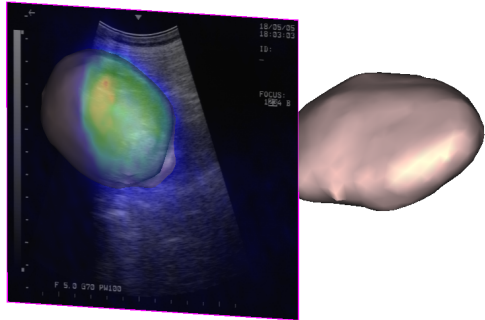

(b)

Fig. 3. (a) 3D SPECT-2D US registration. (b) The US image is displayed in 3D with respect to the segmented kidneys.

physician does the US exam directly after the SPECT acquisition and the image registration is performed on the fly (cf. Fig. 3a). Each US image is also displayed in $3 \mathrm{D}$ with respect to the segmented surfaces of the kidneys (cf. Fig. 3 b).

\section{Results}

We assessed the accuracy of the system using a plate comprising a grid of runnels. The runnels are filled with Technetium and the SPECT of the grid is acquired. Then, without moving the grid, we use a calibrated pointer equipped with a rigid body to locate in $B E D$ the centers of the runnels. The resulting point cloud is registered to the SPECT data using our algorithm. The error is computed by measuring the distance between the points and the reconsctructed runnels in SPECT. Five grid acquisitions were performed with different SPECT acquisition parameters. The overall registration error computed over the resulting 1092 points is mean $=1.5 \mathrm{~mm}, \max =6.3 \mathrm{~mm}$ and $R M S=2.0 \mathrm{~mm}$. The US calibration accuracy, on the other hand, is given by the software implementing the freehand membrane scan method. The calibration error, computed over 240 segmented points, is $\max =2.6 \mathrm{~mm}$ and $R M S=1.0 \mathrm{~mm}$.

Although a global registration error could not be assessed due to the difficulty to design a SPECT and US compatible phantom, we think that the combined errors from SPECT calibration and optical localisation, on the one hand, along with the US calibration errors, on the other hand, are compatible with the intended use of the system i.e. use the functionnal information to guide the sonographical exploration of the patient's kidney.

Automatic kidney segmentations were successfully carried out on 5 patients and the registration proved to have, in all cases, clinically relevant accuracy throughout the exploration field. We rejected 1 exam where obvious patient 
movements have led to permanent (i.e. not breathing related) registration misalignement.

\section{Discussion}

From the practical point of view, the registration procedure is fast and easy to perform. It is also low-cost since it does not require MRI imaging or 3D sonography. The extra time required for the installation of the system is about 5 to 10 minutes. The only time consuming calibration procedures are performed only once and without the need for patient interaction. The fact that the SPECT and US exams are done at the same time in the same room might be considered as a drawback. This requires an extra effort of organization from the clinical point of view although it saves a lot of time for the patient.

The kidneys undergo deformations and displacements while we breathe 16. After a 30 min SPECT acquisition the patient might also feel the need to move. Any such movement breaks our hypothesis for rigid relationship between patient and bed and introduces error. Nevertheless, due to the duration of the acquisition, kidney respiratory movements are also present within the SPECT volume and although we might imagine strategies to stick to a reproductible position such as deep inspiration or deep expiration, as suggested in [16], we will not be able to overcome the problem from the SPECT side. An alternative way would be to consider that the SPECT data contains information about the activity of the organ in some 'mean' position which could be recovered with breath monitoring. But we didn't explore further this possibility. Another source of motion are the interactions between the physician and the patient such as US probe pressure on the back of the patient. The physician must take care not to apply excessive pressure on the probe during the exam.

The scope of the system can also be extended. For example we can imagine a renal puncture guidance using US enhanced with SPECT information. Still assuming rigid registration, which of course is not the exact reality, we can also imagine applications of this registration strategy to the sentinel node biopsy in patients with breast cancer, in which case the traditionally US guided protocol could benefit from the SPECT registration.

\section{Acknowledgements}

Authors wish to thank César Jiménez and Andrés Pérez from the nuclear medicine service, as well as Dr. Reginesi from radiology service, for their help and constant advice throughout the research process. This project has been financially supported by the FONDEF D01-I-1035 (Chile), the Alfa IPECA European project and carried out in collaboration with Praxim-Medivision (France) and Koelis (France). 


\section{References}

1. Maintz, J., Viergever, M.: A survey of medical image registration. Medical Image Analysis 2(1), 1-36 (1988)

2. Walimbe, V., Zagrodsky, V., Raja, S., Jaber, W.A., DiFilippo, F.P., Garcia, M.J., Brunken, R.C., Thomas, J.D., Shekhar, R.: Mutual information-based multimodality registration of cardiac ultrasound and spect images: a preliminary investigation. International Journal of Cardiovascular Imaging 19(6), 483-494 (2003)

3. Ellis, R.E., Toksvig-Larsen, S., Marcacci, M., Caramella, D., Fadda, M.: A biocompatible fiducial marker for evaluating the accuracy of ct image registration. In: Lemke, H.U., Vannier, M.W., Inamura, K., Farman, A.G. (eds.) Computer assisted radiology, Excerpta medica - international congress series, vol. 1124, pp. 693-698 (1996)

4. Peters, T., Davey, B., Munger, P., Comeau, R., Evans, A., Olivier, A.: Threedimensional multimodal imageguidance for neurosurgery. IEEE Transactions on medical imaging 15(2), 121-128 (1996)

5. Kagadis, G.C., Delibasis, K.K., Matsopoulos, G.K., Mouravliansky, N.A., Asvestas, P.A., Nikiforidis, G.C.: A comparative study of surface- and volume-based techniques for the automatic registration between ct and spect brain images. Med. Phys. 29, 201-213 (2002)

6. Wolfsberger, S., Rossler, K., Regatschnig, R., Ungersbock, K.: Anatomical landmarks for image registration in frameless stereotactic neuronavigation. Neurosurg. Rev., Department of Neurosurgery, University of Vienna Medical School 25(1-2), 8-72 (2002)

7. Pluim, J.P., Maintz, J.B., Viergever, M.A.: Mutual-information-based registration of medical images: a survey. IEEE Transactions on Medical Imaging 22(8), 9861004 (2003)

8. Studholme, C., Hill, D.L.G., Hawkes, D.J.: Automated 3-d registration of $\mathrm{mr}$ and ct images of the head. Medical Image Analysis 1(2), 163-175 (1996)

9. Peria, O., Chevalier, L., Francois-Joubert, A., Caravel, J.P., Dalsoglio, S., Lavallee, S., Cinquin, P.: Using a $3 \mathrm{~d}$ position sensor for registration of spect and us images of the kidney. In: Ayache, N. (ed.) CVRMed 1995. LNCS, vol. 905, pp. 23-29. Springer, Heidelberg (1995)

10. Lango, T.: Ultrasound guided surgery: Image processing and navigation. Thesis, Norwegian University of Science and Technology (2000)

11. McInerney, T., Terzopoulos, D.: Deformable models in medical image analysis: A survey. Medical Image Analysis 1(2), 91-108 (1996)

12. Delingette, H.: General object reconstruction based on simplex meshes. INRIA, Sophia-Antipolis, France, Tech. Rep. 3111 (1997)

13. Delingette, H.: Simplex meshes: A general representation for 3-d shape reconstruction. INRIA, Sophia-Antipolis, France, Technical Report 2214 (1994)

14. Montani, C., Scateni, R., Scopigno, R.: Discretized marching cubes. In: IEEE Visualization, Proceedings of the conference on Visualization 1994, Washington D.C., pp. 281-287 (1994)

15. Galdames, F.J., Perez, C.A., Estévez, P.A., Held, C.M.: Segmentation of renal spect images based on deformable models. In: SURGETICA 2005, Computer-Aided Medical Interventions: tools and applications, pp. 89-96 (2005)

16. Schwartz, L., Richaud, J., Buffat, L.: Kidney mobility during respiration. Radiother. Oncol. 32(1), 84-86 (1994) 